A. Berti ${ }^{1,2}$, M.R. Yacoub ${ }^{1}$, P. Stahl Skov ${ }^{3}$, S. Falkencrone ${ }^{3}$, L. Casati ${ }^{2}$, S. Burastero ${ }^{4}$, M.G. SABBADINI ${ }^{1,2}$, G. COLOMBO ${ }^{1,2}$

\title{
Histamine release positive test associates with disease remission in chronic spontaneous urticaria: a proof-of-concept study
}

\author{
${ }^{1}$ Department of Allergy and Clinical Immunology, IRCCS San Raffaele Scientific Institute, Milan, Italy \\ ${ }^{2}$ Vita-Salute San Raffaele University, IRCCS San Raffaele Scientific Institute, Milan, Italy \\ ${ }^{3}$ University Hospital of Southern Denmark, Odense, Denmark \\ ${ }^{4}$ Unit of Leukocyte Biology, DIBIT, IRCCS San Raffaele Scientific Institute, Milan, Italy
}

\section{KEY WORDS}

chronic urticaria; CSU; histamine release test; autologous serum skin test; autologous plasma skin test

\section{Corresponding author}

Alvise Berti

Department of Allergy and Clinical Immunology

IRCCS San Raffaele Scientific Institute

Via Olgettina 60, 20132 Milan, Italy

Phone: +3902 26433468

Fax: +3902 26434301

E-mail: berti.alvise@hsr.it,

alvise.berti@gmail.com

Doi

10.23822/EurAnnACI.1764-1489.17

\begin{abstract}
Summary
Background. Histamine release (HR) test has previously been shown to predict the presence of endogenous histamine-releasing factors in chronic spontaneous urticaria (CSU). Objectives and methods. Twenty CSU patients unresponsive to antihistamine treatment were enrolled in order to evaluate the correlations between HR test results and demographic features, quality of life, disease activity, clinical course, and autologous serum and plasma skin tests (ASST and APST). Results. All patients with positive HR test (9/9, 100\%) had a more severe disease activity at onset (urticaria activity score, UAS > 2) when compared to negative HR test patients $(5 / 11 ; p=0.04)$. Quality of life questionnaires' results were not substantially different between $H R$ positive and negative subgroups at baseline ( $p>0.05)$, and results of HR test and ASSTI APST did not co-segregate $(p>0.05)$. After 12 months, patients with a positive HR test had a significant reduction of disease activity $(p=0.003)$ whereas patients with a negative HR test did not $(p>0.05)$, leading to disease remission and antihistamine treatment withdrawal in $67 \%$ (6/9) of positive HR test patients versus 18\% (2/11) of negative HR test patients ( $p=$ 0.027). Conclusions. Positive HR test may predict spontaneous CSU remission at 12 months.
\end{abstract}

\section{Introduction}

Chronic urticaria $(\mathrm{CU})$ is defined by the presence of urticaria occurring daily or almost daily for more than 6 weeks (1). Overall, CU persists for more than 1 year in $70 \%$ of patients affected, and in almost $15 \%$ of cases it lasts for 5 years (2). Chronic spontaneous urticaria (CSU) refers more specifically to those form of $\mathrm{CU}$ not caused by vascular inflammation (urticarial vasculitis) or triggered by an identifiable external factor, including physical stimuli, and inherited or acquired inflammatory diseases. Approximately $30-50 \%$ of cases of CSU are thought to have an autoimmune etiology, primarily correlated to the presence of $\operatorname{IgG}$ autoantibodies directed against FcERI $\alpha$ receptor or less commonly IgE, that leads to mast cells degranulation (1-5). The demonstration of these autoantibodies is not usually part of common clinical practice. Hence, a surrogate in vivo test called autologous serum skin test (ASST) has been demonstrated to be a reasonably predictive clinical test to indicate the presence of these histamine-releasing functional autoantibodies $(6,7)$. Their presence has already been shown to be associated with a more severe disease (2,3), but not with disease duration (6). Moreover, some authors noted that autologous plasma skin test (APST) causes 
wheal-and-flare reactions much more frequently than ASST, and observed an increase of thrombin generation in plasma of patients with CSU, suggesting a mechanism of mast cells degranulation different from autoantibody-mediated activation (8).

Histamine is the most important mediator in CU which is released upon stimulation by both circulating basophils and tissue-infiltrating mastocytes $(1,3)$. Functional assay of histamine release (HR) from healthy non atopic donors' basophils incubated with CU patients' serum has previously been shown to have a good sensitivity and specificity for endogenous histamine-releasing factors presence, either antibodies or not (9). However, the relationship between the ability of CSU patients' serum to induce HR, and patients' demographics, disease activity, quality of life, clinical course and ASST/APST has never been studied before.

\section{Material and methods}

\section{Patients and study design}

We conducted an observational and prospective study on 20 adult CSU patients unresponsive to antihistamine medications. All forms of chronic urticaria other than CSU were ruled out by medical history and by diagnostic work up, according to European Academy of Allergy and Clinical Immunology (EAACI) guidelines (1,5). Patients diagnosed with CSU, who had persistence of daily symptoms (daily presence of at least one itchy wheal) despite a course of at least 30 days of antihistamine therapy (at least a single dose of anti-H1 antihistamine daily), were enrolled. Antihistamine therapies allowed for the enrolled patients were cetirizine $10 \mathrm{mg}$, desloratadine $5 \mathrm{mg}$, loratadine $10 \mathrm{mg}$, bilastine $20 \mathrm{mg}$, ebastine $10 \mathrm{mg}$. After the 30-day trial period, patients were treated for the following year according to EAACI guidelines. A dose of antihistamine up to 4 times was allowed. A short course of steroid (prednisone $25 \mathrm{mg}$ tapered in 5-7 days) in order to induce initial remission and or to recover from eventual exacerbations was allowed, and based on clinical judgment. Ant leukotriene agents were also allowed. At the time of patients enrollment, omalizumab was not approved in Europe for the treatment of CSU. Treatments were tapered and then stopped in those patients without CSU signs and symptoms for at least 30 days. Following informed consent, each participant underwent a complete clinical assessment during the first visit in our tertiary care center. Patients were then monitored for 12 months and treated with symptomatic treatment for CSU according to physician's judgment, which typically included a daily single dose of a second-generation H1-antihistamine, a daily double antihistamine dose or a daily double dose plus an anti-leukotriene agent, according to disease severity.

\section{In vivo and in vitro tests}

Venipuncture and ASST/APST were performed after the withdrawal of antihistamine treatment for at least 72 hours. Patients under- went in vivo assessment of ASST and APST as previously described $(6,8)$ and in vitro determination of HR from healthy subjects' basophils incubated with patients serum. For HR test, white blood cells containing 1-2\% basophils from a healthy non-atopic donor were incubated with patients' sera in the presence of interleukin (IL)-3. Histamine was measured by the glass fiber method, as previously described (9). Cut-off value for HR was set to $>16.5 \%$. In order to avoid conscious or unintended unconscious bias, HR test results were masked to clinicians taking care of the patients (GC, MNY, $\mathrm{AB}, \mathrm{LC}$ ) until the end of the 1-year observational period, while clinical data were masked to laboratory operators (PSS, SF, SB) as well.

\section{Disease activity and quality of life assessment}

Disease activity was assessed by Urticaria Activity Score (UAS) at 0 and 12 months. Daily UAS values (0-6 points) were calculated on the patient's conditions over the previous 24 hours, by adding the score value for number of wheals (0-3) and for pruritus intensity (0-3), as already described (10). Patients quality of life was evaluated at baseline by Chronic Urticaria Quality Of Life Questionnaire (CU-Q2oL) and other metrics commonly used in clinical practice (Visual Analogic Scale, VAS; Dermatology Life Quality Index, DLQI) (1,10-14).

\section{Statistical analysis}

Variable values were presented as median and range for serological and immunological biomarkers, unless otherwise specified. Categorical variables were compared using the Fisher's exact test. Normally and non-normally distributed variables were compared using the Student's t-test and Mann-Whitney U-test, respectively. Treatment efficacy in each group was assessed by means of Wilcoxon Signed Rank test (paired) analysis. GraphPad Prism version 6.00 was utilized for statistical analysis. A p-value $<0.05$ was considered statistically significant.

\section{Results}

\section{Study cohort}

Seventeen $(85 \%)$ patients were female, mean age was 42 (range, 18-67). Angioedema associated with CSU in 70\% (14/20) of cases. Autoimmune thyroid dysfunction (altered thyroid-stimulating hormone [TSH] serum levels with autoantibodies against thyroglobulin and/or thyroperoxidase and/or TSH-receptor) was present in 35\% of cases (6 patients with hypothyroidism, one patient with hyperthyroidism), 5\% (1/20) had isolated autoantibodies anti-thyroglobulin and thyroperoxidase antibodies without thyroid dysfunction, 20\% (4/20) had antinuclear antibody (ANA) positivity without any autoimmune condition, and 5\% (1/20) had Sjögren's syndrome. Clinical and demo- 
Table 1 - Demographic features of patients $(n=20)$.

\begin{tabular}{ll}
\hline Feature & Number or $\%$ \\
\hline Age & 42 years (range 18-67) \\
\hline Sex & $85 \%$ female, $15 \%$ male \\
\hline Disease duration & 3.5 years (range $0.5-16)$ \\
\hline Associated angioedema & $70 \%$ \\
\hline Autoimmune disease (different from thyroiditis) & $5 \%$ (1 patient with Sjögren's syndrome) \\
\hline Thyroid disfunction $^{1}$ & $35 \%$ (1 Basedow-Graves' disease; 6 Hashimoto' disease) \\
\hline Isolated thyroid autoimmunity $^{2}$ & $5 \%$ \\
\hline Antinuclear antibody (ANA) & $20 \%$ \\
\hline
\end{tabular}

${ }^{1}$ Autoimmune thyroid dysfunction was defined as an altered thyroid-stimulating hormone (TSH) serum levels with autoantibodies against thyroglobulin and/or thyroperoxidase and/or TSH-receptor.

${ }^{2}$ Isolated thyroid autoimmunity was defined as a normal thyroid-stimulating hormone (TSH) serum levels with autoantibodies against thyroglobulin and/or thyroperoxidase and/or TSH-receptor.

graphic features are summarized in table 1. Quality of life questionnaires' results were not substantially different between HR positive and negative groups at baseline (table 2).

\section{HR test results and ASST/APST results do not cosegregate}

Sixty percent $(12 / 20)$ of patients scored positive for both ASST and APST, 5\% (1/20) scored positive for only ASST, and 20\% (4/20) scored positive for only APST. Around 15\% (3/20) scored negative for both ASST and APST (figure 1A).

HR test was positive (i.e., > 16.5\%) in 45\% (9/20) of patients. Notably, patients with positive test had a highly significant increase $\%$ of HR compared to those who scored negative ( $\mathrm{p}<$ 0.001; figure 1B).

We compared in vivo (ASST/APST) and in vitro (HR) test results, but they did not co-segregate (figure 1C; $\mathrm{p}>0.05$ in all comparisons). In particular, $67 \%(6 / 9)$ and $55 \%$ (6/11) of patients with positive and negative HR test results respectively, scored positive for both ASST and APST. Eleven percent (1/9) and 27\% (3/11) of patients with respectively positive and negative HR test results scored positive for APST only, while 9\% (1/11) of patients with negative HR scored positive for ASST only (figure 1C). Thyroid dysfunction or autoimmune conditions did not associate with any HR or ASST/APST subset ( $\mathrm{p}>0.05$, respectively).

\section{Patients with a positive HR test have a more severe disease at onset}

All patients with positive HR test $(9 / 9,100 \%)$ had a more severe disease activity at onset (UAS score $>2$ ), compared to approximately $45 \%(5 / 11)$ of patients with HR negative test ( $\mathrm{p}=0.04$; figure $1 \mathrm{D})$. During the 1-year observation period, all the patients were treated following EAACI guidelines and according to clinical judgment, in order to obtain CSU remission or the best control of the disease. Thirty-five percent (7/20) of patients received a single second-generation $\mathrm{H} 1$-antihistamine daily dose, $35 \%$ (7/20) received a double antihistamine daily dose and 30\% (6/20) received a double antihistamine daily dose plus an antileukotriene agent. Overall, 8 patients stopped therapies because of the resolution of signs and symptoms, while 3 patients used antihistamines only when needed ( $\leq 3 /$ week) because of a frank improvement of the clinical picture (table 3 ).

Table 2 - Quality of life questionnaires'scores (Dermatology Life Quality Index [DLQI]; Chronic Urticaria Quality OfLife Questionnaire [CU-Q2oL], Visual Analogic Scale [VAS]) in patients with positive and negative HR test results at baseline. Comparison between HR positive and negative patients'scores were non significant ( $p>0.05)$ for each questionnaire used.

\begin{tabular}{ccc}
\hline Questionnaire & HR positive patients & HR negative patients \\
\hline DLQI (with range) & $11(1-22)$ & $10(1-19)$ \\
\hline CU-Q2oL (with range) & $43(16-76)$ & $44(23-61)$ \\
\hline VAS (with range) & $65(50-91)$ & $59(40-85)$ \\
\hline
\end{tabular}


Figure 1 - (A) In vivo test: autologous serum skin test (ASST) and autologous plasma skin test (APST) in our cohort of patients ( $n=20)$. (B) In vitro test: histamine release (HR) from healthy nonatopic donor basophils incubated with CSU patients serum. HR test was positive (i.e., > 16.5\%) in 9/20 patients and negative in 11/20 patients ( $p<0.001) ; H C=$ healthy controls. (C) Comparison between in vivo and in vitro test results: there was no correlation and the results of the two tests did not co-segregate. The solid spots represent the patients with $H R$ positive and negative test results respectively (in vitro test), while the two intersecting sets represent the ASST and APST results (in vivo test). (D) Comparison between HR test results and severity of disease assessed by urticaria activity score (UAS) at baseline: HR test positivity significantly associates with a more severe disease (UAS score $>2 ; p=0.04$ ).

$1 \mathrm{~A}$

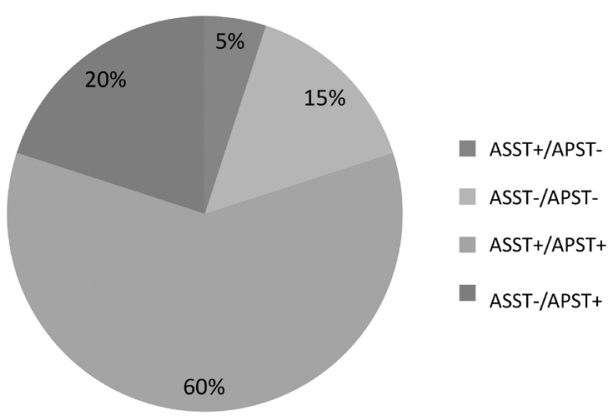

$1 C$



$1 B$

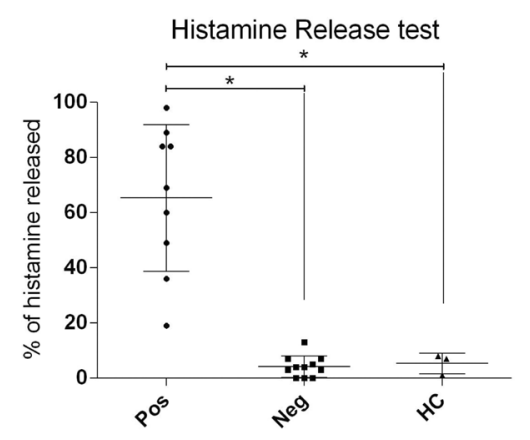

1D

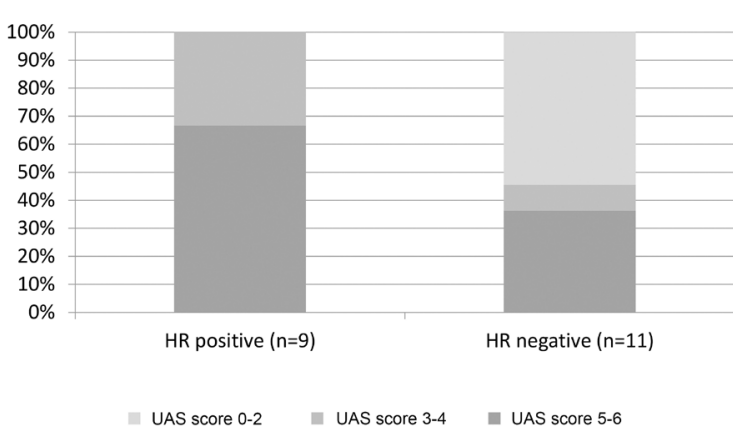

Table 3 - Antihistamine treatment withdrawn in patients with HR positive and negative test after 12 months.

\begin{tabular}{ccc}
\hline Antihistamine & HR positive patients & HR negative patients \\
\hline Daily & $33 \%(3 / 9)$ & $55 \%(6 / 11)$ \\
\hline When needed & $0 \%$ & $27 \%(3 / 11)$ \\
\hline Withdrawal $^{*}$ & $67 \%(6 / 9)$ & $18 \%(2 / 11)$ \\
\hline
\end{tabular}

* Statistically significant $(\mathrm{p}<0.05)$

A positive HR test result at baseline predicts spontaneous CSU remission and associates with antihistamine treatment withdrawal at 1 year

Patients were monitored for 12 months; patients with a positive HR test had a significant reduction of disease activity assessed by UAS after 12 months ( $\mathrm{p}=0.003$; figure $2 \mathbf{A}$ ), whereas pa- tients with a negative HR test did not ( $p>0.05$; figure $2 B$ ). When comparing patients who had an improvement in UAS in the observation period versus those who did not, a trend in favor of patients with positive HR test was observed, since 78\% (7/9) and 45\% (5/11) reported positive and negative HR test respectively $(\mathrm{p}=0.197)$. 
Figure 2 - Disease severity variations after 12 months of symptomatic therapy in patients with HR positive test results $(A)$ and patients with HR negative test results (B). Disease activity was assessed by Urticaria Activity Score (UAS) based on the patient's conditions over the previous 12 hours. In the box, median UAS (with range; min-max values) at onset and after 12 months.

A

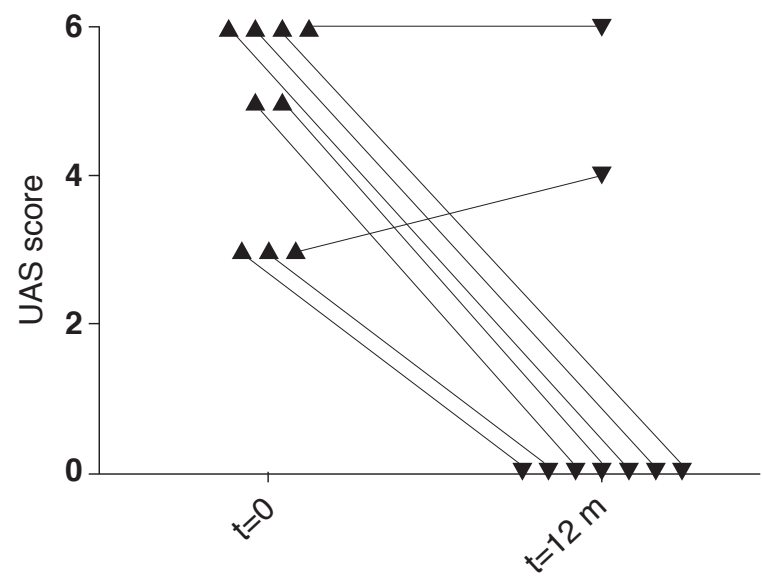

\begin{tabular}{|l|l|l|}
\hline Median UAS & $5(3,6)$ & $0(0,6)$ \\
\hline
\end{tabular}

Moreover, 67\% (6/9) of patients with positive HR test were able to completely withdraw therapy and achieve complete CSU remission compared to $18 \%(2 / 11)$ of patients with a negative HR test results ( $\mathrm{p}=0.027$; table 3$)$. No statistical difference in the median UAS reduction after 12 months between patients who scored positive for both ASST/APST, negative for both ASST/ APST, and positive for only APST was observed ( $p>0.05$ in all comparisons; table 4).

\section{Discussion}

Our results suggest that positive HR test associates with two apparently contrasting phenotypic characteristics of antihista-
B HR test negative pts

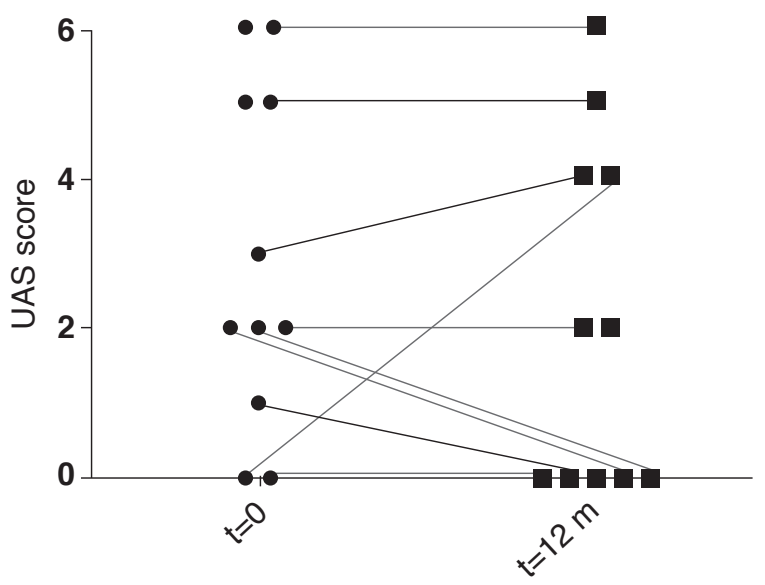

\begin{tabular}{|l|l|l|}
\hline Median UAS & $2(0,6)$ & $2(0,6)$ \\
\hline
\end{tabular}

mine-unresponsive CSU, i.e., a more severe disease at onset (as depicted by a UAS score $>2$; figure 1D) and a more frequent spontaneous disease remission at 12 months. Patients with a positive HR test had a higher reduction of disease activity ( $\mathrm{p}$ $=0.003)$ and withdrew antihistamine treatment in a higher number of cases $(p=0.027)$ when compared to patients with a negative HR test results, suggesting that HR test may predict disease remission.

Although the HR test does not inform on the underlying pathological mechanisms of CSU, we could speculate that patients' sera with negative HR test (approximately half of the patients) need to interact with one or more other cellular or humoral

Table 4 - Median UAS score stratified for in vivo results at onset and at 12 months. No significant difference between $t=0$ and $t=12$ was found within each group ( $p>0.05$ ).

\begin{tabular}{lccc}
\hline Subsets & Patients & $\mathbf{t}=\mathbf{0}$ & $\mathbf{t}=\mathbf{1 2}$ \\
\hline ASST/APST +/+ (range) & $\mathrm{n}=12$ & $4(0-6)$ & $2(0-6)$ \\
\hline ASST/APST +/- (range) & $\mathrm{n}=1$ & 0 & 0 \\
\hline ASST/APST -/+ (range) & $\mathrm{n}=4$ & $4(2-6)$ & $2(0-5)$ \\
\hline ASST/APST -/- (range) & $\mathrm{n}=3$ & $2(0-6)$ & 0 \\
\hline
\end{tabular}


factors to induce basophils and mast cells activation, possibly expressed in skin and target tissues. Along this line, the subset of CSU patients with positive HR test could be characterized by a yet to be defined systemic component, which in the same time would be per se capable to induce both a relatively more severe urticaria symptoms and its exhaustion in a year time.

A potential limitation of this study is that UAS score for the assessment of CSU severity could not necessarily indicate a true disease remission, but may simply reflect disease fluctuations. Unfortunately, we did not use UAS calculated on 7 days (UAS7) because the recruitment of patients studied preceded the latest EAACI guidelines for CSU (16), which suggested the use of UAS7 instead of UAS. Of note, two third of those with positive HR test achieved CSU complete remission after 12 months leading to antihistamine therapy withdrawn, compared with only $18 \%$ of patients with a negative HR test results $(p=0.027)$. Since by definition patients studied complained daily symptoms for at least 30 days before enrollment, this represents a meaningful result and indicates, together with the UAS reduction after 12 months, that HR may be a genuine predictor of remission in patients unresponsive to antihistamine therapy.

One of the novelty of our study is that we analyzed the relationship between in vivo and in vitro tests, and we did not find any association. Using the ASST as the true outcome for HR test validation, its sensitivity and specificity for CU was reported to be around $75 \%$ (9). Taken altogether, HR test may have a complementary role to ASST/APST, helping clinician in the diagnosis if the in vivo skin tests provide doubtful results or if they cannot be assessed.

Interestingly, we did not find any significant associations between quality of life and HR test outcome, probably because the individual perception of CSU interference varies considerably among different patients (15).

Several authors suggested that patients with CSU who scored positive for APST only may be less responsive to conventional therapy (8). Notably, the majority of patients of our cohort who scored positive for APST only were HR test negative (3/4, 75\%; figure $1 \mathrm{C}$ ). In order to evaluate whether the poor response to treatment of HR negative patients could be segregated in this subset of patients, we stratified patients for ASST/APST results and compared median UAS before and after 12 months of therapy. We found that UAS reduction was similar between patients who scored positive for both ASST/APST, APST alone, and patients who scored negative for both ASST/APST, suggesting that HR test independently predicts treatment response. An in vitro test could likely predict outcome of CSU more precisely than an in vivo test. At variance with the in vitro test, the interpretation skin testing is operator-dependent, and sometimes and its reproducibility is linked to the physician expertise.
Prior reports with basophil activation test (BAT; consisting of sera of CSU patients incubated with resting basophils of normal donors) induce histamine release and upregulate CD63 and CD203c $(22,23)$. BAT with CD63 may have a role in assessing severity of disease and predicting outcome, but results are not completely homogeneous. (22-24) Although a direct comparison has not been published, HR test instead is easy to perform and interpret, and results can be easily standardized between different centers (9). No statistical association of autoimmune thyroid dysfunction (or isolated thyroid autoimmunity) with HR or APST/ASST results was observed in our cohort. The mutual relationship of these two conditions is well known and accounts for 10 to $27 \%$ of cases in literature $(17,18,19)$. In our cohort the percentage of thyroid disease is slightly greater, perhaps as a consequence of a selection bias of those patients with an antihistamine resistant CSU. However, this may also depend on small sample size, which clearly represents a limit for this and other subgroups' analysis in our study. This study has a good internal validity because a very select homogeneous group (only CSU excluding inducible or physical urticaria; only patients daily symptomatic after at least a single dose of antihistamine therapy for at least 1 month) is chosen. Given that CSU is likely a group of diseases instead a single one, likely with different predisposing factors, disease mechanisms and perhaps different results to in vivo and in vitro tests, we chose a very selected group characterized by the unresponsiveness to conventional doses of symptomatic drugs. This gives us the chance to draw precise conclusions about the persistence of disease on our sample albeit the small size, but at the same time it impacts the generalizability of our results, since our study sample represents the patients still symptomatic despite conventional treatment doses instead of the whole CSU population.

In conclusion, HR test seems to predict CSU spontaneous remission at 12 months in patient with persistent disease, since patients with positive HR test underwent therapy withdrawal in a higher number of cases as compared to those with negative results. Even if larger studies to confirm these preliminary data are needed, HR test could be helpful in discriminating at onset those CSU patients who are more likely to benefit from antihistamine therapy from those who may need more aggressive treatment options (20-21).

\section{References}

1. Zuberbier T, Asero R, Bindslev-Jensen C, Canonica GW, Church MK, Gimenez-Arnau A, et al. EAACI/GA(2)LEN/EDF/WAO guideline: definition, classification and diagnosis of urticaria, Allergy. 2009;64(10):1417-26.

2. Toubi E, Kessel A, Avshovich N, Bamberger E, Sabo E, Nusem $\mathrm{D}$, et al. Clinical and laboratory parameters in predicting chronic urticaria duration: a prospective study of 139 patients. Allergy. 2004;59(8):869-73. 
3. Sabroe RA, Fiebiger E, Francis DM, Maurer D, Seed PT, Grattan $\mathrm{CE}$, et al. Classification of anti-FcepsilonRI and anti-IgE autoantibodies in chronic idiopathic urticaria and correlation with disease severity. J Allergy Clin Immunol. 2002;110(3):492-9.

4. Konstantinou GN, Asero R, Maurer M, Sabroe RA, Schmid-Grendelmeier P, Grattan CE. EAACI/GA(2)LEN task force consensus report: the autologous serum skin test in urticaria. Allergy. 2009;64(9):1256-68.

5. Konstantinou GN, Asero R, Ferrer M, Knol EF, Maurer M, Raap $\mathrm{U}$, et al. EAACI taskforce position paper: evidence for autoimmune urticaria and proposal for defining diagnostic criteria. Allergy. 2013;68(1):27-36.

6. Sabroe RA, Grattan CE, Francis DM, Barr RM, Kobza Black A et al. The autologous serum skin test: a screening test for autoantibodies in chronic idiopathic urticaria. Br J Dermatol. 1999;140(3):446-52.

7. Sabroe RA, Greaves MW. Chronic idiopathic urticaria with functional autoantibodies: 12 years on. $\mathrm{Br} \mathrm{J}$ Dermatol. 2006;154(5):813-9.

8. Asero R, Tedeschi A, Riboldi P, Cugno M. Plasma of patients with chronic urticaria shows signs of thrombin generation, and its intradermal injection causes wheal-and-flare reactions much more frequently than autologous serum. J Allergy Clin Immunol. 2006;117(5):1113-7.

9. Platzer MH, Grattan CE, Poulsen LK, Skov PS. Validation of basophil histamine release against the autologous serum skin test and outcome of serum induced basophil histamine release studies in a large population of chronic urticaria patients. Allergy. 2005;60(9):1152-6.

10. Młynek A, Zalewska-Janowska A, Martus P, Staubach P, Zuberbier T, Maurer M. How to assess disease activity in patients with chronic urticaria? Allergy. 2008;63(6):777-80.

11. O'Donnell BF, Lawlor F.,Simpson J, Morgan M, Greaves MW. The impact of chronic urticaria on the quality of life. Br. J. Dermatol 1997;136(2):197-20112. Baiardini, M. Pasquali, F. Braido, F. Fumagalli, L. Guerra, E. Compalati, M. et al. A new tool to evaluate the impact of chronic urticaria on quality of life: chronic urticaria quality of life questionnaire (CU-QoL). Allergy. 2005;60(8): 1073-8.

13. Loo W. J., Diba V., Chawla M., Finlay A. Y. Epidemiology and Health Services Research Dermatology Life Quality Index : influence of an illustrated version. Br J Dermatol. 2003;148(2):279-84.
14. Basra A. M. K., Fenech R., Gatt R. M., Salek M. S.,. Finlay A. Y. The Dermatology Life Quality Index 1994- 2007: a comprehensive review of validation data and clinical results. Br J Dermatol. 2008;159(5):997-1035.

15. Baiardini I, Giardini A, Pasquali M, Dignetti P, Guerra L, Specchia C, et al. Quality of life and patients' satisfaction in chronic urticaria and respiratory allergy. Allergy. 2003;58(7):621-3.

16. Zuberbier T, Aberer W, Asero R, Bindslev-Jensen C, Brzoza Z, Canonica GW et al. The EAACI/GA(2) LEN/EDF/WAO Guideline for the definition, classification, diagnosis, and management of urticaria: the 2013 revision and update. Allergy. 2014;69(7):868-87.

17. Verneuil L, Leconte C, Ballet JJ, Coffin C, Laroche D, Izard JP et al. Association between chronic urticaria and thyroid autoimmunity: a prospective study involving 99 patients. Dermatology. 2004;208(2):98-103.

18. Confino-Cohen R, Chodick G, Shalev V, Leshno M, Kimhi O, Goldberg A. Chronic urticaria and autoimmunity: associations found in a large population study. J Allergy Clin Immunol. 2012;129(5):1307-13.

19. Sánchez-Borges M, Caballero-Fonseca F, Capriles-Hulett A. Subtypes of chronic urticaria in patients attending allergy clinics in Venezuela. Eur Ann Allergy Clin Immunol. 2014;46(6):210-5.

20. Cordeiro Moreira AS, Rosmaninho Lopes de Soares E Silva MI, Pereira Guilherme MA, da Silva Ferreira JA, Fonseca Moreira da Silva JP. Use of omalizumab in the treatment of chronic urticaria. Eur Ann Allergy Clin Immunol. 2016;48(6):242-6.

21. Greiwe J, Bernstein JA. Therapy of antihistamine-resistant chronic spontaneous urticaria. Expert Rev Clin Immunol. 2016;3:1-8.

22. Wedi B, Novacovic V, Koerner M, KappA. Chronic urticaria serum induces histamine release, leukotriene production, and basophil CD63 surface expression-inhibitory effects of anti-inflammatory drugs. J Allergy Clin Immunol. 2000;105:552-60.

23. Yasnowsky KM, Dreskin SC, Efaw B, Schoen D, Vedanthan PK, Alam R et al. Chronic urticaria sera increase basophil CD203c expression. J Allergy Clin Immunol. 2006;117(6):1430-4.

24. Szegedi A, Irinyi B, Gal M, Hunyadi J, Danko K, Kiss E et al. Significant correlation between the CD63 assay and the histamine release assay in chronic urticaria. Br J Dermatol. 2006;155(1):67-75. 